\title{
Psicopatologia na Infância: \\ Levantamento Epidemiológico
}

Child Psychopathology: Epidemiologic Study

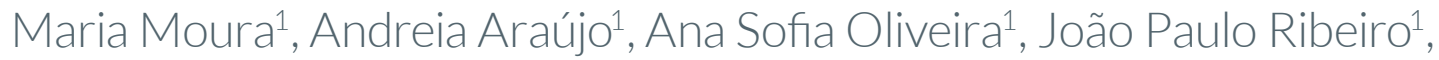
Catarina Rosa ${ }^{1}$

Autor Correspondente:

Maria Moura: [maria.moura@hvfx.pt]

Estrada Nacional n 1, Povos 2600-009, Vila Franca de Xira, Portugal

\section{RESUMO}

INTRODUÇÃO: As perturbações psiquiátricas da infância podem persistir durante a adolescência e vida adulta, vindo a ter um impacto negativo e que pode afetar o desenvolvimento e a autonomia do futuro adulto. O presente estudo epidemiológico surgiu da necessidade de conhecer a probabilidade de psicopatologia na infância, na população do primeiro ciclo do concelho de Vila Franca de Xira. Um dos objetivos é permitir, de forma sustentada, aferir as necessidades reais, do ponto de vista da Saúde Mental, da população infantil deste concelho.

MATERIAL E MÉTODOS: Participaram neste estudo encarregados de educação e professores de 3063 crianças das escolas do primeiro ciclo do concelho, através do preenchimento do Strengths and Difficulties Questionnaire - SDQ. O tratamento estatístico dos dados foi realizado através do software IBM ${ }^{\circledR}$ SPSS ${ }^{\circledR}$ (IBM - Statistical Package for the Social Sciences, versão 21).

RESULTADOS: A taxa de resposta aos questionários distribuídos foi de 82,27\% nos encarregados de educação e 86,15\% nos professores. A probabilidade de existência de psicopatologia nos questionários preenchidos pelos encarregados de educação e pelos professores foi semelhante (respetivamente 14,2\% e 13,68\%). Não se encontraram diferenças significativas entre a probabilidade de psicopatologia entre géneros.

DISCUSSÃO E CONCLUSÃO: Os dados reportam uma probabilidade de psicopatologia que vai ao encontro da prevalência mundial de 12,36\%, nas idades abrangidas por este estudo. Este tipo de levantamento epidemiológico é essencial no alerta para políticas de promoção da saúde que assegurem as necessidades na área da saúde mental infantil. Destaca a necessidade de prevenção e intervenção atempada nesta área.

PALAVRAS-CHAVE: Criança; Inquéritos e Questionário; Perturbações do Comportamento Infantil/epidemiologia; Perturbações do Comportamento Infantil/psicologia; Psicometria 


\section{ABSTRACT}

INTRODUCTION: PSychiatric disorders presented during childhood may persist during adolescence and adulthood, and have a negative impact that may affect the development and autonomy of the future adult. The present epidemiological study arose from the need to know the probability of psychopathology in childhood, in the population of the elementary schools of the county of Vila Franca de Xira. One of the goals is to allow, in a sustained way, to assess the real needs from the point of view of Mental Health of the young children of this county.

MATERIALS AND METHODS: Caregivers and teachers of 3063 children from elementary schools of the county participated in this stud through the filling of the Strengths and Difficulties Questionnaire (SDQ). The statistical treatment of the data was performed through IBM ${ }^{\circledR}$ SPSS ${ }^{\circledR}$ (IBM - Statistical Package for the Social Sciences, version 21).

RESULTS: The response rate was $82.27 \%$ in the caregivers and $86.15 \%$ in the teachers. The probability of existence of psychopathology in the questionnaires filled out by caregivers and teachers was similar (14.2\% and 13.68\%). No significant differences were found between the probability of psychopathology between genders.

DISCUSSION AND CONCLUSION: The data report a probability of psychopathology that matches the world prevalence of $12.36 \%$, at the ages covered by this study. This type of epidemiological survey is essential for the alert in health promotion policies that assure children's mental health needs. It highlights the need for timely prevention and intervention in this area.

KEYWORDS: Child; Child Behavior Disorders/epidemiology; Child Behavior Disorders/psychology; Psychometrics; Surveys and Questionnaires

\section{INTRODUÇÃO}

Os problemas de saúde mental na infância e adolescência têm aumentado significativamente nas últimas décadas, ${ }^{1}$ constituindo no presente um foco de investigação, não só por poderem prejudicar o desenvolvimento da criança (com impacto no rendimento escolar e nas relações sociais), mas também por se encontrarem associados ao risco de desenvolver problemas psicossociais na vida adulta, constituindo assim um dos grandes desafios de Saúde Pública a nível mundial. ${ }^{2}$

De acordo com a Organização Mundial de Saúde (OMS) Região Europeia, uma em cada cinco crianças apresenta evidências de problemas mentais, proporção essa que tende a aumentar. Destas crianças, cerca de metade sofre de uma perturbação psiquiátrica. ${ }^{3}$ Uma intervenção inexistente ou inadequada favorece a ocorrência de situações graves na vida adulta. ${ }^{4}$

Estudos epidemiológicos como o presente têm como um dos objetivos primordiais, apresentar evidências da necessidade e reforçar a importância da disponibilização de recursos para a área da Saúde Mental Infanto-Juvenil, pois ainda que seja notória a prevalência de problemas de comportamento e emocionais, apenas uma pequena percentagem destas crianças e jovens entra em contacto com Serviços de Saúde Mental. ${ }^{5}$

O conhecimento de prevalência de problemas mentais, assim como o estudo da probabilidade de psicopatologia na infância, poderá ser um importante auxiliar na prevenção e no planeamento dos serviços de saúde dispensados à comunidade. ${ }^{4}$ Uma meta-análise recente estima que o predomínio de problemas de saúde mental nas idades supracitadas alcança os 12,36\% a nível mundial. ${ }^{6}$

A identificação de desvios significativos no desenvolvimento e comportamento infantil poderá ocorrer primeiramente na escola. Os professores revelam-se, portanto, como fonte de informação de enorme relevância na avaliação de situações de disfunção psicossocial, tornando-se imperativo explorar as suas perceções na avaliação de determinados comportamentos das crianças.?

A criança existe num contexto sociocultural e, não havendo determinismo genético a nível psicopatológico, há vários fatores de risco descritos na emergência de dificuldades ao nível do desenvolvimento psicoafetivo da infância. Estes podem ser intrínsecos, e relacionados com o temperamento e genética da própria criança, assim como extrínsecos, relacionados com o ambiente sociofamiliar da criança. Salienta-se a artificialidade desta dicotomia, uma vez que os estudos de epigenética vieram demonstrar como o ambiente codetermina a expressão génica, numa influência recíproca gene/ambiente. A patologia na infância existe quando emergem formas de funcionamento desadaptativas e causadoras do sofrimento. ${ }^{8}$

As primeiras relações criam um padrão de expectativas na relação da criança consigo e com os outros, os vários contextos podem potenciar certos traços de funcionamento em detrimento de outros. $\mathrm{O}$ Homem é intrinsecamente marcado pelo contexto em que nasce e se desenvolve. Fala-se de psicopatologia infantil quando há menor flexibilização na capacidade da criança lidar com 
a adversidade e quando emerge sofrimento psíquico.

Existe uma diferença entre géneros ao nível da expressão e incidência de psicopatologia na infância. Estas diferenças podem dever-se ao facto de os dois géneros poderem estar sujeitos a diferentes fatores de risco ambientais, distintos níveis de um mesmo risco ambiental e ter diferentes mecanismos de resposta (marcados por aspetos biológicos distintos entre géneros, diferenças essas que podem ser diferencialmente amplificadas ou diminuídas entre indivíduos). A elevada consciência social de crianças do género feminino em comparação com o género masculino traduz-se ao nível da empatia, das habilidades sociais, da culpabilidade após transgressões e da capacidade de compreensão dos outros e das suas intenções. ${ }^{9}$ Tendo em conta estas particularidades em conjunto com a maturação mais precoce e com as habilidades de linguagem do género feminino, seria de esperar que as raparigas fossem mais resilientes para os problemas. O género feminino usa estratégias de enfrentamento emocional e rumina sobre os problemas enquanto o masculino se apoia na distração e em estratégias mais agressivas.

A magnitude das diferenças entre géneros varia com a idade, o que sugere que muitos processos influenciam diferencialmente os níveis de comportamentos no género masculino e feminino durante o curso do desenvolvimento. Revisões extensas de literatura sugerem que a ansiedade de separação e fobias específicas têm uma idade de início mais precoce e são os distúrbios de ansiedade mais diagnosticados em crianças. ${ }^{10}$ Os distúrbios de ansiedade são geralmente mais comuns no sexo feminino. As perturbações de comportamento e as perturbações do neurodesenvolvimento (perturbações do espectro do autismo, problemas ao nível do desenvolvimento da linguagem, perturbação de hiperatividade e défice de atenção) têm idades de início muito precoces e apresentam-se em números muito superiores no género masculino. Em contraste, as perturbações precoces da adolescência, como a depressão e ansiedade, têm uma predominância no género feminino. ${ }^{11}$ Assim, é de esperar que no início da idade escolar seja o género masculino o mais afetado por perturbações mentais, ao passo que com a passagem para a adolescência se assiste a uma inversão desta tendência, com o género feminino a ser mais afetado.

A importância do estudo das diferenças entre géneros relativamente à predominância da psicopatologia tem ganho relevância nos últimos tempos. Problemas previamente ignorados em um dos géneros, porque ocorrem com menos frequência, como por exemplo o comportamento antissocial no género feminino e a depressão no masculino, são agora alvo de uma maior atenção. ${ }^{12}$ Coloca-se também a questão de como os fatores sociais ao longo dos tempos podem condicionar estas diferenças, não só ao nível das características dos géneros feminino e masculino, como também ao nível da expressão psicopatológica do sofrimento mental.

Na sociedade atual têm vindo a emergir mudanças familiares onde se atenuam os contrastes de papéis femininos e masculinos. "Atribuir à mulher o papel de cuidar do lar e ao homem o de sair à rua para prover o sustento da família não só é um modelo arcaico que remonta às origens do processo civilizatório como soa hoje em dia como um estereótipo tangenciando o ridículo" (Osorio, 1996). ${ }^{13}$ Esta equivalência dos papéis femininos e masculinos parece gerar identificações nos descendentes que viabilizam modelos nos quais as diferenças entre géneros se encontram esbatidas. Por outro lado, como refere Gilles Lipovetsky, a atual "sociedade do vazio", que se estrutura em torno de um egocentrismo esvaziado de pensamento, promove o agir sem pensar e, consequentemente, as patologias do agir. ${ }^{14}$

Perante as mudanças sociofamiliares descritas, torna-se relevante analisar indicadores de psicopatologia na infância ao nível da comunidade. $\bigcirc$ presente estudo é um estudo epidemiológico de rastreio da probabilidade de psicopatologia na infância permitindo, não só esta análise, mas também, de forma mais sustentada, aferir as necessidades reais da população infantil da área de Vila Franca de Xira.

Esta iniciativa surge no seguimento da abertura da Consulta de Pedopsiquiatria no Hospital de Vila Franca de Xira, no sentido de delinear o perfil desta população e das suas eventuais necessidades clínicas.

\section{MATERIAL E MÉTODOS}

O presente estudo é de caráter quantitativo e transversal. Os dados foram recolhidos a partir de um universo constituído por todas as escolas do $1^{\circ}$ ciclo de Vila Franca de Xira. Participaram neste estudo encarregados de educação e professores de 3063 crianças, de ambos os géneros, a frequentar qualquer um dos anos escolares. Pais e professores preencheram assim um questionário a fim de obter informações relativas a características comportamentais e emocionais das crianças. No presente estudo foi utilizado o Questionário de Capacidades e Dificuldades (Strengths and Difficulties Questionnaire-SDQ), instrumento constituído por 25 itens que tem como objetivo monitorizar a probabilidade de problemas psiquiátricos na população infanto-juvenil em cinco áreas, gerando scores de sintomas emocionais, 
problemas de comportamento, hiperatividade/desatenção, problemas entre pares e problemas nos comportamentos pro-sociais que são somados para gerar uma pontuação total de dificuldades. O questionário incluiu um suplemento que avalia o impacto das dificuldades no contexto sociofamiliar (questionando se correspondente pensa que a criança apresenta um problema relevante, e, em caso afirmativo, inquire adicionalmente sobre angústia global, prejuízo social, sobrecarga e cronicidade).

O questionário pode ser aplicado aos professores e encarregados de educação de crianças dos 4 aos 16 anos de idade e ao inquirido é pedido que baseie a sua resposta no comportamento dos últimos seis meses da criança. ${ }^{15}$

O SDQ permite uma boa descriminação entre crianças com e sem sintomas psiquiátricos e há evidências que indicam que pode ser usado como medida de rastreio de perturbações psiquiátricas em amostras comunitárias. ${ }^{16}$ A especificidade do SDQ é de 94,6\% e a sensibilidade de 63,3\%.17 O SDQ foi traduzido em mais de 40 idiomas e encontra-se validado em Portugal. ${ }^{18}$

Assim, o Hospital de Vila Franca de Xira com a Equipa de Pedopsiquiatria, a Direção do ACES Estuário do Tejo e a Câmara Municipal de Vila Franca de Xira promoveram, junto das Escolas Primárias do concelho de Vila Franca de Xira, um inquérito à população escolar.

A Câmara Municipal agilizou o processo e efetuou os contactos com as Escolas expondo a investigação. Após todos os Encarregados de Educação e Professores terem sido informados da investigação foi facultado a estes e aos professores de crianças do $1^{\circ}$ ciclo o Questionário de Capacidades e Dificuldades (SDQ), tendo sido assegurada a confidencialidade e o uso sigiloso da informação recolhida.

O tratamento dos questionários foi anónimo, e ficou a cargo e sob a responsabilidade da Equipa de Pedopsi- quiatria do Hospital de Vila Franca de Xira. O tratamento estatístico dos dados recolhidos foi realizado através do software IBM ${ }^{\circledR}$ SPSS ${ }^{\circledR}$ (IBM - Statistical Package for the Social Sciences, versão 21).

Foi realizada uma análise descritiva dos resultados obtidos em cada uma das escalas de avaliação, assim como do suplemento de impacto, considerando as frequências absolutas e respetivas percentagens. Para comparação de diferenças entre sexos, escolaridade e escola, foram utilizados os testes da binominal e de aderência do Qui-quadrado. Para verificar se existem diferenças na probabilidade de psicopatologias entre cada uma das psicopatologias analisadas recorreu-se ao teste de Friedman. A associação entre a probabilidade de existência de psicopatologia e o género, escolaridade e escola que frequentam as crianças foram analisados com recurso ao teste Qui-quadrado. Por fim, também se compararam os resultados obtidos pelos professores e pais numa análise de concordância tendo-se recorrido para o efeito ao teste de McNemar. $\bigcirc$ valor de significância escolhido para análise das relações entre estas variáveis foi de 5\% ( $p<0,05)$, sendo que os resultados obtidos inferiores a este valor são alusivos da existência de uma associação ou diferenças estatisticamente significativas.

\section{RESULTADOS}

Do total de inquéritos fornecidos aos encarregados de educação a taxa de resposta foi de 82,27\% ( $n=2520$, do total de 3063). Do total de questionários fornecidos aos professores a taxa de resposta foi de 86,15\% ( $n=2639$, do total de 3063).

Verifica-se que não existem diferenças significativas entre a proporção de crianças do género masculino e feminino. Existem diferenças significativas entre os vários anos nos dados dos encarregados de educação e professores ( $x 2=23,09$ e $\times 2=23,61, p=0,000)$.

TABELA 1. Caracterização sociodemográfica dos questionários preenchido pelos encarregados de educação e pelos Professores (F= frequência absoluta).

\begin{tabular}{|c|c|c|c|c|c|}
\hline & & \multicolumn{2}{|c|}{ Encarregados de Educação } & \multicolumn{2}{|c|}{ Professores } \\
\hline \multicolumn{2}{|c|}{ Dados sociodemográficos } & $F$ & $\%$ & $F$ & $\%$ \\
\hline \multirow{5}{*}{ Ano Escolaridade } & $1^{\circ}$ Ano & 630 & 25,00 & 605 & 22,93 \\
\hline & $2^{\circ}$ Ano & 541 & 21,47 & 608 & 23,04 \\
\hline & $3^{\circ}$ Ano & 638 & 25,32 & 667 & 25,27 \\
\hline & $4^{\circ}$ Ano & 711 & 28,21 & 759 & 28,76 \\
\hline & Total & 2520 & & 2639 & \\
\hline \multirow{4}{*}{ Género } & Feminino & 949 & 37,66 & 1028 & 39,95 \\
\hline & Masculino & 894 & 35,48 & 1016 & 38,49 \\
\hline & Desconhecido & 677 & 26,87 & 595 & 22,55 \\
\hline & Total & 2529 & & 2639 & \\
\hline
\end{tabular}


A possibilidade de psicopatologia na amostra de inquéritos fornecidos aos encarregados de educação é de 26,3\% ( $n=665)$ e de 22,93\% ( $n=605)$ na amostra de inquéritos fornecidos aos professores, valores que poderão ser sobre inclusivos. Contudo, se aumentarmos a especificidade da análise - analisando apenas os casos de elevada probabilidade de psicopatologia (correndo o risco de aumentar a taxa falsos negativos), temos respetivamente uma probabilidade de $14,2 \%$ ( $n=359)$ e de $13,68 \%(n=361)$.

Analisando apenas os casos onde há uma elevada probabilidade de psicopatologia, podemos observar que a hiperatividade é a patologia com maior probabilidade de ocorrência ( $n=594,23,57 \%$ ), seguindo-se os problemas emocionais ( $n=409,16,23 \%$ ), os problemas de comportamento ( $n=369,14,64 \%$ ), os problemas de relacionamento com os pares ( $n=309,12,26 \%$ ) e, por fim, os problemas nos comportamentos pro-sociais ( $n=1,1,23 \%$ ). Existem diferenças estatisticamente significativas entre estes vários problemas ( $\times 2 f=924,82, p=0,00)$ de acordo com o teste de Friedman (Fig. 1).

Nos questionários dos professores, especificando o tipo de patologia, denota-se maior probabilidade de ocorrência de problemas de hiperatividade ( $n=593$, $22,47 \%$ ), seguindo-se os problemas comportamentais ( $n=416,15,76 \%$ ) e, em seguida, com menor expressão, os problemas emocionais ( $n=183,6,93 \%$ ) (Fig. 2). As diferenças entre os resultados obtidos nas várias psico-
NORMAL

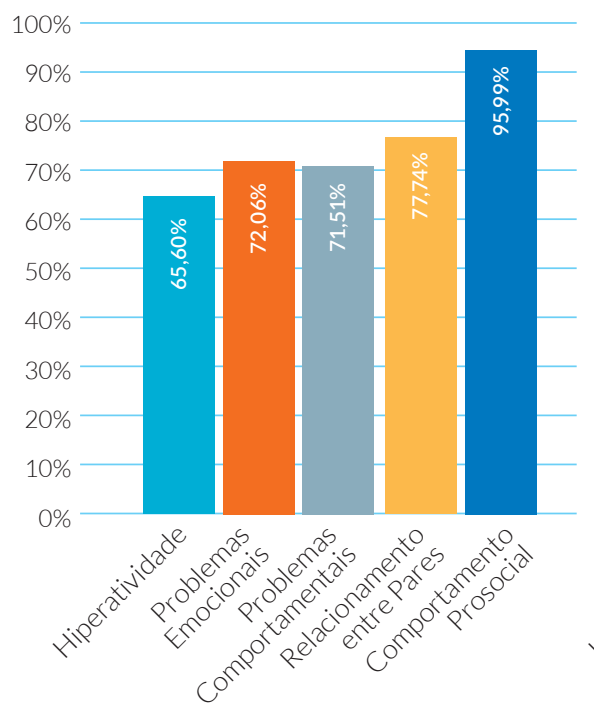

POSSÍVEL

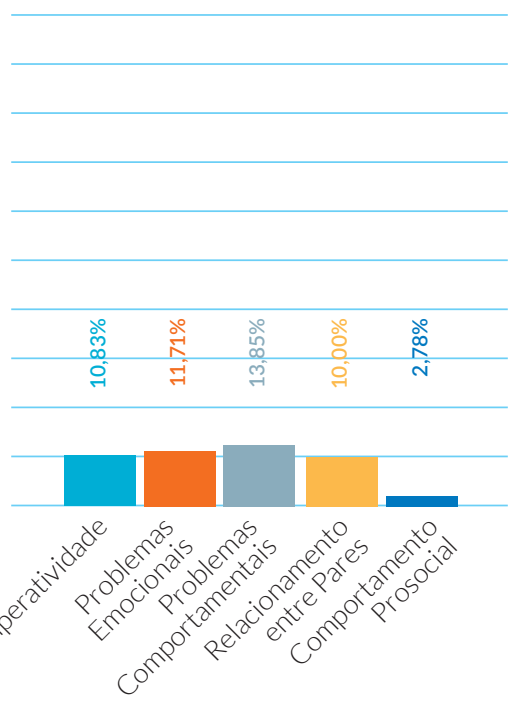

PROVÁVEL

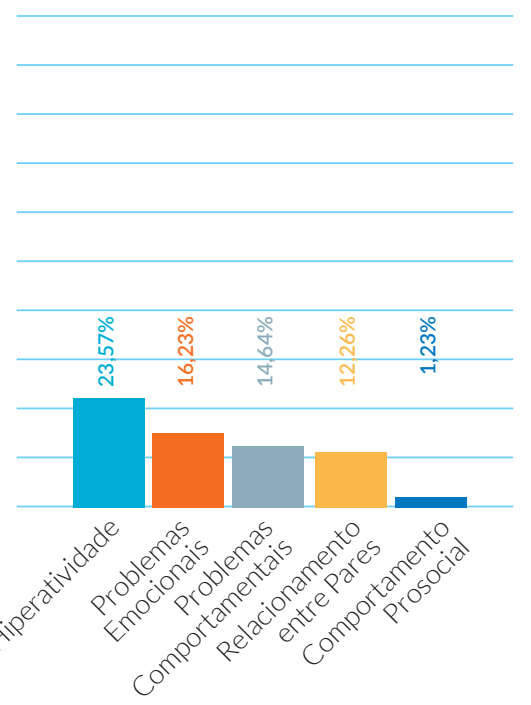

FIGURA 1. Probabilidade de psicopatologia na amostra de inquéritos fornecidos aos encarregados de educação por categorias de problemas.

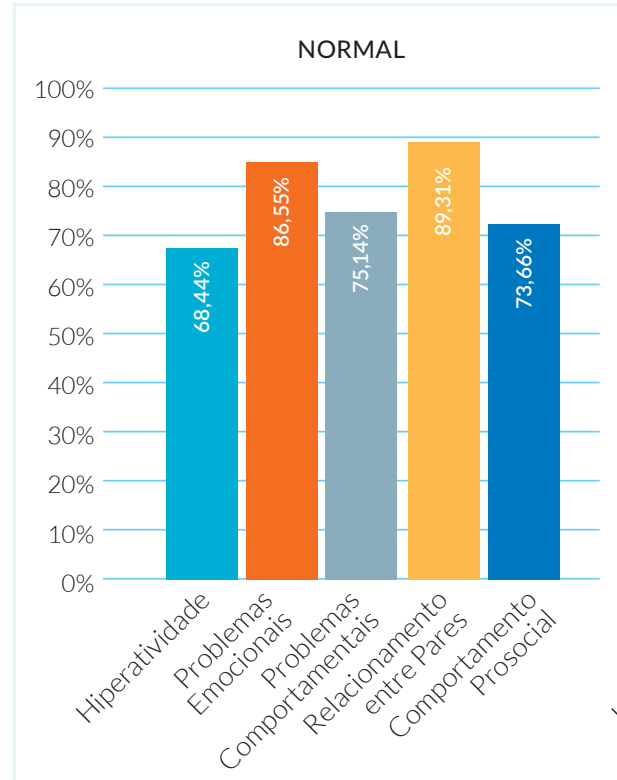

POSSÍVEL

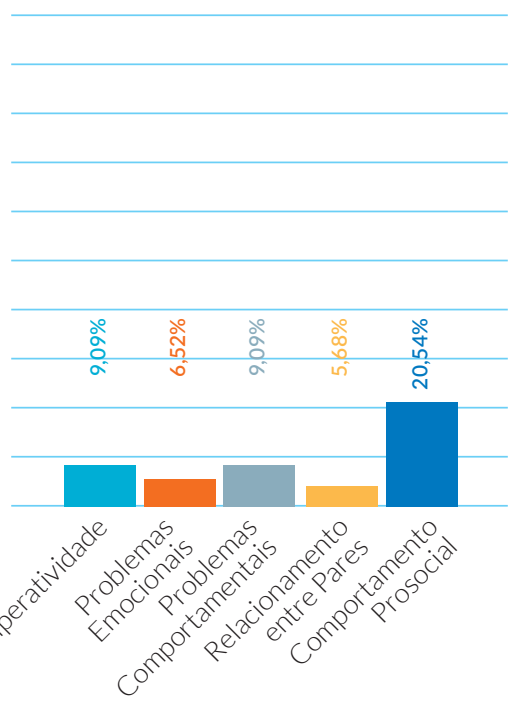

PROVÁVEL

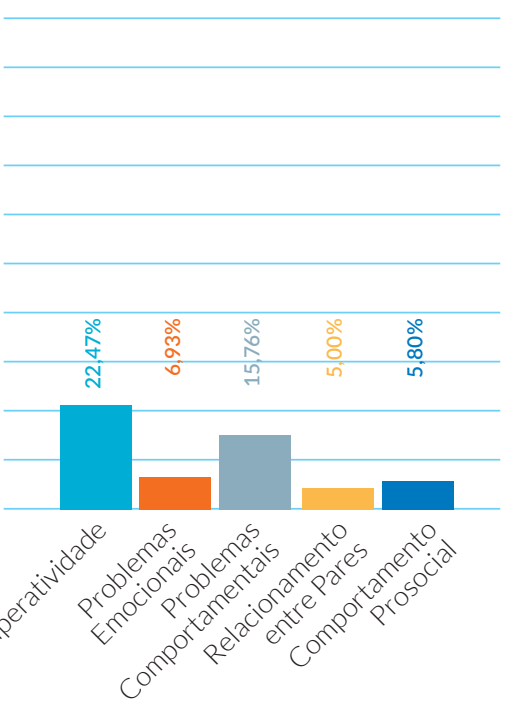

FIGURA 2. Probabilidade de psicopatologia na amostra de inquéritos fornecidos aos professores por categorias de problemas. 
patologias são estatisticamente significativas $(659,44$, $p=0,000)$.

Na análise por géneros não se verificam diferenças significativas no que se refere a probabilidade de psicopatologia em geral nos dados dos encarregados de educação e professores ( $\times 2=1,36^{i}$ e $\times 2=2,11^{i i}, p=0,51$ ), sendo a probabilidade de ambos os sexos terem algum tipo de psicopatologia muito semelhante.

Da análise pelas diferentes escolas não se encontram diferenças estatisticamente significativas ao nível de probabilidade de psicopatologia.

Relativamente à análise dos resultados por ano escolar, segundo os dados dos encarregados de educação, registam-se diferenças significativas na probabilidade de ocorrência de psicopatologia ( $x 2=17,31, p=0,008$ ), sendo que os alunos com psicopatologia do $2^{\circ}$ (91/541 $1^{i i}$, $16,82 \%)$ e do $3^{\circ}$ ano (110/638iv, 17,24\%) se encontram em maior proporção. Relativamente aos problemas comportamentais parece haver maior probabilidade de ocorrência no $2^{\circ}$ ano de escolaridade (96/17,75\%, x2 = $17,16, p=0,009)$.

Estas diferenças não se verificam nos dados dos professores por ano de escolaridade ( $x 2=12,40, p=0,06$ ), no que se refere à probabilidade de psicopatologia em geral. No entanto, quando se analisam os dados por categorias de problemas, verifica-se que no que respeita à probabilidade de ocorrência de problemas de relacionamento interpessoal existe uma associação significativa com o ano de escolaridade ( $x 2=18,14, p=0,00)$, com maior probabilidade no $3^{\circ}$ ano (44/667, 6,60\%).
Em relação ao suplemento de impacto, 1822 (72,30\%) dos 2520 questionários preenchidos pelos encarregados de educação, se encontravam preenchidos. A probabilidade de impacto nesta amostra é de 22,23\% ( $n=$ 405). No grupo de questionários preenchidos pelos professores a taxa de resposta ao suplemento de impacto da psicopatologia foi de 73,36\%, o que corresponde a 1936 dos 2639 questionários. Quanto ao impacto, a sua probabilidade de ocorrência é de 17,46\% ( $n=338)$.

Conforme se pode observar na Figura 3, podemos verificar que dos 1822 encarregados de educação que respondem à questão do impacto que a psicopatologia provoca, a maioria refere que os filhos têm problemas de hiperatividade ( $n=529,29,03 \%$ ), seguindo-se os problemas emocionais ( $\mathrm{n}=352,19,32 \%$ ) e os problemas comportamentais ( $n=330,18,11 \%$ ). No grupo dos 1936 questionários dos professores (Fig. 4), há uma maior probabilidade de hiperatividade ( $n=539$, $27,84 \%$ ), mas segue-se uma maior probabilidade de problemas comportamentais ( $n=369,19,06 \%$ ), e só depois de problemas emocionais ( $n=166,8,57 \%$ ). De acordo com o teste de Friedman as diferenças verificadas são estatisticamente significativas $(p=0,00)$.

Existe uma associação significativa entre a probabilidade de psicopatologia e a probabilidade de impacto dessa mesma psicopatologia tanto na amostra de encarregados de educação como de professores ( $x 2=466,05$ e $x$ $2=596,59, p=0,00$ ), sendo superior no grupo que considera que existe maior probabilidade de psicopatologia (213/339i e 221/338ii, 62,83\% e 65,38\%).

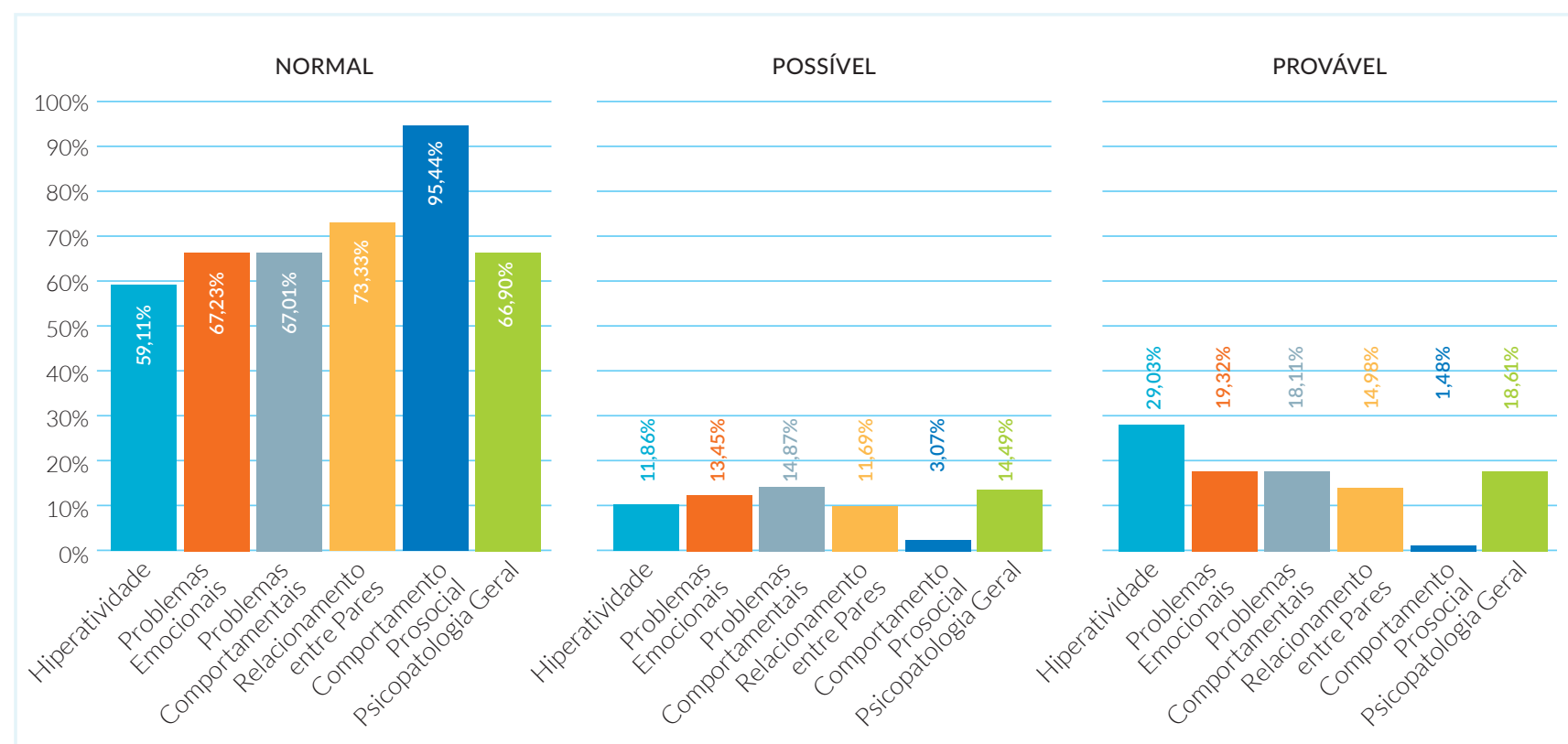

FIGURA 3. Resultados do preenchimento do suplemento de impacto (encarregados de educação) com especificação por categorias de problemas. 

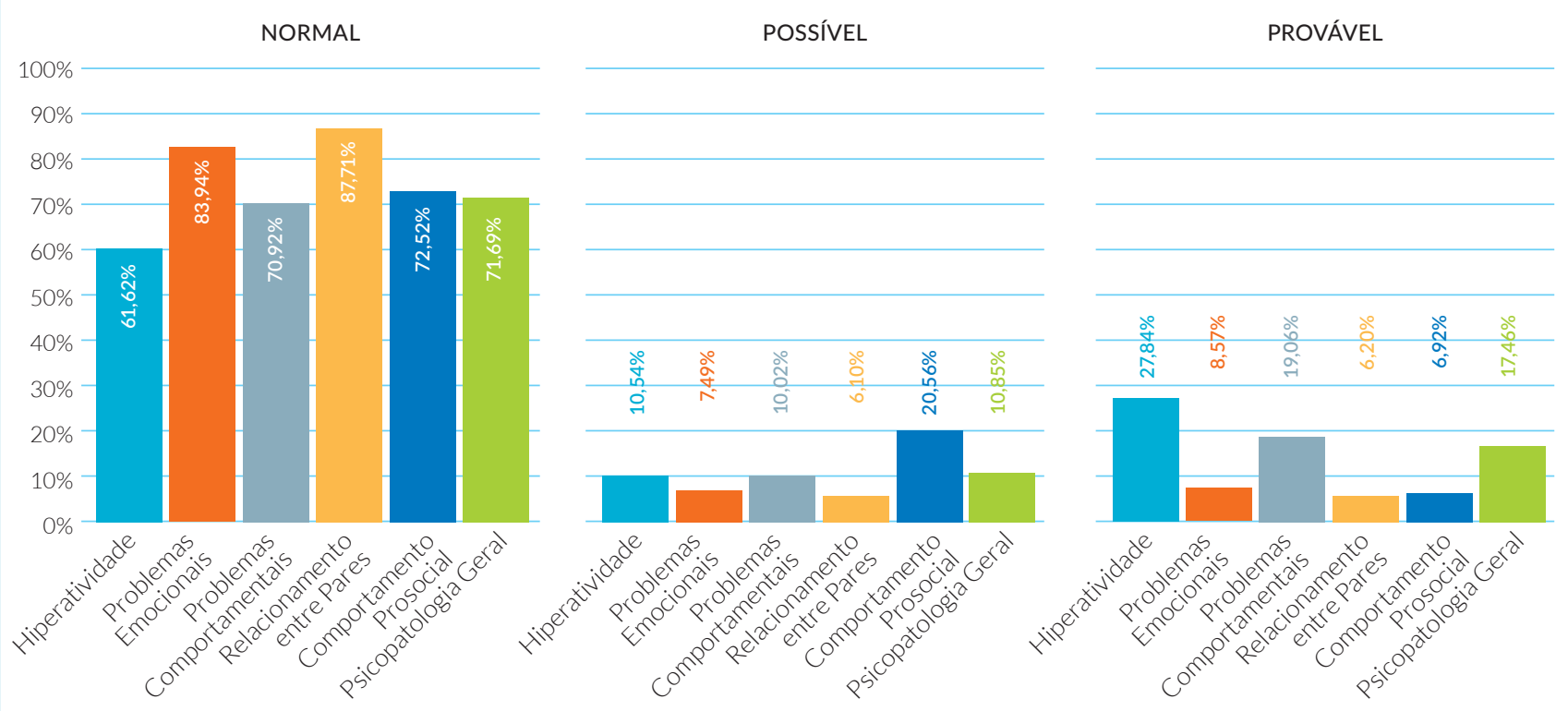

FIGURA 4. Resultados do preenchimento do suplemento de impacto (professores) com especificação por categorias de problemas.

Quanto ao impacto que a psicopatologia apresenta, notamos que não existem diferenças entre os dois géneros tanto na amostra de encarregados de educação como de professores ( $\times 2=0,37, p=0,83$ e $\times 2=1,35, p=0,51)$, nem entre $\mathrm{o}$ ano de escolaridade $(x 2=10,48 p=0,10 \mathrm{e}$ $x 2=7,45, p=0,28$.

Ao nível da análise de concordância entre encarregados de educação e professores (teste de McNemar), a probabilidade de psicopatologia de acordo com os encarregados de educação ( $n=359,14,25 \%)$ é superior à verificada pelos professores ( $n=305,12,10 \%)$.

Há uma concordância entre professores e encarregados de educação quanto à probabilidade de ocorrência de hiperatividade, assim como quanto à probabilidade de ocorrência de problemas de comportamento e de impacto.

Relativamente à probabilidade de ocorrência de problemas emocionais não existe concordância entre professores e encarregados de educação, e a probabilidade de problemas emocionais de acordo com os encarregados de educação ( $n=409,16,24 \%)$ é superior à verificada pelos professores ( $n=64,2,54 \%)$. Este último grupo engloba situações que passam mais despercebidas a nível escolar por serem menos visíveis e perturbadoras do funcionamento das aulas.

\section{DISCUSSÃO E CONCLUSÃO}

A taxa de resposta aos questionários distribuídos foi considerável, podendo afirmar-se que existiu uma boa colaboração dos encarregados de educação e dos professores no presente estudo epidemiológico (82,27\% dos encarregados de educação e 86,15\% dos professores).

A probabilidade de existência de psicopatologia nos questionários preenchidos pelos encarregados de educação e pelos professores foi bastante semelhante (14,2\% para encarregados de educação e 13,68\% para professores). Salienta-se que estes dados vão de encontro aos valores apresentados por uma recente meta-análise onde se fala duma prevalência mundial de 13,4\% de perturbações mentais em crianças e adolescentes e duma prevalência mundial de 12,36\% nas idades abrangidas por este estudo. ${ }^{6}$

Nos questionários preenchidos pelos encarregados de educação, naqueles em que existe probabilidade de psicopatologia, a hiperatividade é o problema com maior probabilidade de ocorrência (23,57\%) seguido de imediato pelos problemas emocionais (16,23\%), estes diferem dos questionários preenchidos pelos professores, onde apesar do problema com maior probabilidade de ocorrência ser a hiperatividade (22,47\%), se seguem de imediato os problemas do comportamento (15,76\%), ficando este a uma distância considerável dos problemas emocionais (6,93\%). 
Tal discrepância pode justificar-se com o facto dos problemas do comportamento, que fazem parte de uma série de comportamentos mais externalizantes, se tornarem mais problemáticos em contexto escolar, passando os problemas emocionais, estes mais internalizantes, mais despercebidos por terem menos impacto na sala de aula. De notar que tanto as perturbações do comportamento como as perturbações emocionais se associam a dificuldades de aprendizagem. ${ }^{19} \mathrm{~A}$ alteração da atenção é um sintoma frequentemente apontado pela escola, onde a necessidade de realização de tarefas que exigem o recurso das funções executivas, nomeadamente a memória de trabalho, colocam a descoberto dificuldades ao nível da focalização atencional, sintoma ubíquo a várias situações, e que causa alerta pela existência de um impacto importante.

Neste estudo não se encontraram diferenças significativas entre a probabilidade de psicopatologia entre géneros. A análise do impacto por género também não revela diferenças estatisticamente significativas. Estes resultados são válidos tanto para os questionários preenchidos pelos encarregados de educação como para os questionários preenchidos pelos professores. Esperaríamos que neste estudo o género masculino tivesse maior probabilidade de psicopatologia quando comparado com o feminino. Porém, e tendo em conta que nas referenciações hospitalares se mantém um predomínio do género masculino (70\%, segundo dados da casuística interna da consulta de Pedopsiquiatria do HVFX, 2016) podemos colocar como hipótese, haver maiores dificuldades de enquadramento do género masculino à "entidade escola", com eventualmente maiores repercussões ao nível da aprendizagem, o que poderá levar a mais referenciações do género masculino aos serviços de saúde mental infantil.

Adicionalmente, por uma questão de sigilo, não foi incluída a data de nascimento das crianças, o que condicionou uma limitação ao nível do conhecimento da idade destas, podendo haver algumas crianças que, não obstante frequentarem o primeiro ciclo, pudessem ter idades acima dos 10 anos. Estes dois aspetos dificultam uma análise mais elucidada das descritas diferenças de psicopatologia por género onde, como já foi explicado, há um predomínio de problemas no género masculino até à puberdade, altura em que o género feminino começa a ser mais representado. ${ }^{12}$

Este tipo de levantamento epidemiológico é essencial no alerta para políticas de promoção da saúde que assegurem as necessidades na área da saúde mental infantil, assente num trabalho multidisciplinar e na constituição de equipas de intervenção alargadas. Os problemas de saúde mental na infância são comuns e prejudicam o rendimento escolar e o relacionamento social. São situações que sem intervenção tendem a persistir na adultícia, podendo favorecer a ocorrência de eventos graves e criando um ciclo que se pode perpetuar na geração seguinte. Torna-se imperativa a necessidade de prevenção e intervenção atempada na área de saúde mental infantil. 20

Será importante salvaguardar que embora o SDQ seja útil para efeitos de despiste, não deverá ser utilizado, de forma isolada, para fazer diagnósticos em saúde mental, uma área de avaliação clínica onde é de extrema importância a integração de informação eventualmente discrepante.

\section{AGRADECIMENTOS}

Robert Goodman, Psiquiatra da Infância e Adolescência, Institute of Psychiatry at the Maudsley, London, UK;

Pedro Caldeira da Silva, Pedopsiquiatra, Chefe da Unidade de Primeira Infância, Hospital Dona Estefânia, Centro Hospitalar de Lisboa Central;

António Nunes e Pedro Bastos, Administradores Executivos do Hospital de Vila Franca de Xira;

Câmara Municipal de Vila Franca de Xira;

Escolas, professores e encarregados de educação que participaram no estudo.

CONFLITOS DE INTERESSE: Os autores declaram não ter qualquer conflito de interesse na realização do presente trabalho.

FONTES DE FINANCIAMENTO: Não houve qualquer fonte de financiamento na realização do presente trabalho.

CONFIDENCIALIDADE DOS DADOS: Os autores declaram ter seguido os protocolos da sua instituição acerca da publicação dos dados de doentes.

PROTEÇÃO DE PESSOAS E ANIMAIS: Os autores declaram que os procedimentos seguidos na elaboração do presente trabalho estão em conformidade com as normas das comissões de investigação clínica e de ética, bem como da declaração de Helsínquia e da Associação Médica Mundial.

CONFLICTS OF INTEREST: The authors declare that they have no conflicts of interest.

FINANCIAL SUPPORT: This work has not received any contribution, grant or scholarship. 
CONFIDENTIALITY OF DATA: The authors declare that they have followed the protocols of their work center on the publication of data from patients.

PROTECTION OF HUMAN AND ANIMAL SUBJECTS: The authors declare that the procedures followed were in accordance with the regulations of the relevant clinical research ethics committee and with those of the Code of Ethics of the World Medical Association (Declaration of Helsinki).

\section{REFERÊNCIAS}

1. Santos M. Problemas de Saúde Mental em Crianças e Adolescentes -Identificar, Avaliar e Intervir. Lisboa: Edições Sílabo; 2013.

2. Ferriolli SHT, Marturano EM, Puntel LP. Contexto familiar e problemas de saúde mental infantil no Programa Saúde da Família. Rev de Saúde Pública. 2007; 41: 251-9.

3. Marques C, Cepêda T. Recomendações para a prática clínica da saúde mental infantil e juvenil nos cuidados de saúde primários. Lisboa: Coordenação Nacional para a Saúde Mental; 2009

4. Fleitlich BW, Goodman R. Epidemiologia. Rev Bras Psiquiatr. 2000; 22 (Supl II): 2-6.

5. Moura M, Encarnação R, Gomes F. O papel do SDQ (Strengths and Difficulties Questionnaire) como medida de Avaliação Psicométrica em Psiquiatria da Infância e Adolescência. Rev APPIA. 2010; 28: 77-88.

6. Polanczyk GV, Salum GA, Sugaya LS, Caye A, Rohde LA. Annual Research Review: A meta-analysis of the worldwide prevalence of mental disorders in children and adolescents. J Child Psychol Psychiatry. 2015; 56: 345-65.

7. Franco V. A psicopatologia infantil vista pelos professores: necessidades de intervenção psicológica em crianças do primeiro ciclo. Rev NUFEN. 2009;1: 105-19.

8. Ramires VR, Passarin, DS, Flores, GG, Santos LG. Fatores de risco e problemas de saúde mental de crianças. Arq Brasil Psicol. 2009; 61:1-14.

9. Brody LR. Gender, emotion, and the family. Cambridge: Harvard University Press; 1999.

10. Kessler RC, Berglund P, Demler O, Jun R, Merikangas KR, Walters EE. Lifetime prevalence and age-of-onset distributions of DSM-IV disorders in the National Comorbidity Survey Replication. Arch Gen Psychiatry. 2005; 62: 593-602.

11. Marcelli D. Infância e Psicopatologia. Lisboa: Climepsi; 2005.

12. Zahn-Waxler C, Shirtcliff EA, Marceau K. Disorders of childhood and adolescence: Gender and psychopathology. Annu Rev Clin Psychol. 2008; 4: 275-303.

13. Osorio LC. Família hoje. Porto Alegre: Artes Médicas; 1996.

14. Lipovetsky G. A era do vazio. Ensaios sobre o individualismo contemporâneo. Lisboa: Editora Relógio d'Água; 1989.

15. Silva TB, Osório FL, Loureiro SR. SDQ: discriminative validity and diagnostic potential. Front Psychol. 2015; 6: 811.

16. Mouris P, Meesters C, Berg F. Strengths and Difficulties Questionnaire (SDQ) - Further evidence for its reliability and validity in a community sample of Dutch children and adolescents. Eur Child Adolesc Psychiatry. 2003; 12: 1-8.

17. Goodman R. The Strengths and Difficulties Questionnaire: a research note. J Child Psychol Psychiatry. 1997; 38: 581-586.
18. Marzocchi GM, Capron C, Di Pietro M, Duran Tauleria E, Duyme M, Frigerio A, et al. The use of the Strengths and Difficulties Questionnaire (SDQ) in Southern European countries. Eur Child Adolesc Psychiatry. 2004; 13 Suppl 2: ||40-46.

19. Marques C. A Saúde Mental Infantil e Juvenil nos Cuidados de Saúde Primários -Avaliação e Referenciação. Rev Port Clin Geral. 2009; 25: 569-75.

20. Fleitlich B, Cortázar PG, Goodman R. Questionário de capacidades e dificuldades (SDQ). Rev Neuropsiquiatr Infanc Adolesc. 2000; 8: 44-50. 